\title{
Interplay of Touschek scattering, intrabeam scattering, and rf cavities in ultralow-emittance storage rings
}

\author{
S. C. Leemann \\ MAX IV Laboratory, Lund University, SE-22100 Lund, Sweden
}

(Received 11 March 2014; published 27 May 2014)

\begin{abstract}
The latest generation of storage ring-based light sources employs multibend achromat lattices to achieve ultralow emittance. These lattices make use of a large number of weak bending magnets which considerably reduces the amount of power radiated in the dipoles in comparison to power radiated from insertion devices. Therefore, in such storage rings, parameters such as emittance, energy spread, and radiated power areunlike 3rd generation storage rings - no longer constant during a typical user shift. Instead, they depend on several varying parameters such as insertion device gap settings, bunch charge, bunch length, etc. Since the charge per bunch is usually high, intrabeam scattering in medium-energy storage rings with ultralow emittance becomes very strong. This creates a dependence of emittance on stored current. Furthermore, since the bunch length is adjusted with rf cavities but is also varied as insertion device gaps change, the emittance blowup from intrabeam scattering is not constant either. Therefore, the emittance, bunch length, and hence the resulting Touschek lifetime have to be calculated in a self-consistent fashion with 6D tracking taking into account not only the bare lattice and rf cavity settings, but also momentary bunch charge and gap settings. Using the MAX IV $3 \mathrm{GeV}$ storage ring as an example, this paper demonstrates the intricate interplay between transverse emittance (insertion devices, emittance coupling), longitudinal emittance (tuning of main cavities as well as harmonic cavities), and choice of stored current in an ultralow-emittance storage ring as well as some implications for brightness optimization.
\end{abstract}

DOI: 10.1103/PhysRevSTAB.17.050705

PACS numbers: 29.20.db, 41.60.Ap, 29.27.Bd, 29.27.Fh

\section{INTRODUCTION}

Although already proposed in the 1990s [1-5], multibend achromat (MBA) lattices have only recently started to appear in storage ring-based light source designs [6-11]. The MBA lattice allows reaching ultralow emittance (i.e., transverse emittances substantially below $1 \mathrm{~nm}$ rad) and presents a path to fully diffraction-limited storage rings for the production of X-rays [12]. When the MAX IV facility $[13,14]$ goes into operation in 2016, its $3 \mathrm{GeV}$ storage ring will become the first ultralow-emittance storage ring based on a MBA $[6,15]$. Its 20-fold lattice employs a 7-bend achromat to achieve $328 \mathrm{pm}$ rad transverse emittance with a circumference of $528 \mathrm{~m}$. As is typical for such ultralow-emittance lattices, the radiative losses in the dipoles $(364 \mathrm{keV} /$ turn) are low compared to what can be expected (roughly $1 \mathrm{MeV} /$ turn) once the ring is fully equipped with insertion devices (IDs) and/or damping wigglers (DWs). This can be recognized in Fig. 1 where various beam parameters are plotted as functions of the number of installed in-vacuum undulators (IVUs). As a consequence, the ring's zero-current emittance at any time depends on the type of installed IDs and their gap

*simon.leemann@maxlab.lu.se

Published by the American Physical Society under the terms of the Creative Commons Attribution 3.0 License. Further distribution of this work must maintain attribution to the author $(s)$ and the published article's title, journal citation, and DOI. settings. As the ID gaps vary during a typical user shift, not only will this change the transverse emittance [16], it willassuming the rf cavities are not adjusted to compensate for gap motion-change the resulting rf acceptance, bunch length, and Touschek lifetime.

In addition, in medium-energy rings the large stored current along with the low transverse emittance leads to very strong intrabeam scattering (IBS) which blows up the beam's $6 \mathrm{D}$ emittance $[17,18]$. Hence, the resulting transverse emittance in ultralow-emittance storage rings at medium energies depends on the stored charge in the bunch. Specifically, the transverse emittance will decrease as the current drops. In state-of-the-art storage rings top-up injection is usually employed to prevent current decay, however, variations of stored charge from bunch to bunch are not uncommon and in certain cases actually desired (e.g., camshaft mode). In such situations, the emittance from bunch to bunch can vary as a function of bunch charge. Even if top-up injection and filling pattern control are used to ensure an even fill, the emittance can still vary as a result of ID gap motion and with it the amount of emittance blowup from IBS. Furthermore, a change of bunch energy spread (as a consequence of e.g., ID gap changes) or bunch length (rf cavity settings, harmonic cavity tuning, or ID gap changes), will also influence IBS and hence the resulting equilibrium emittance. The interplay between IDs, rf cavities, and transverse emittance via IBS will be the subject of Sec. II. 

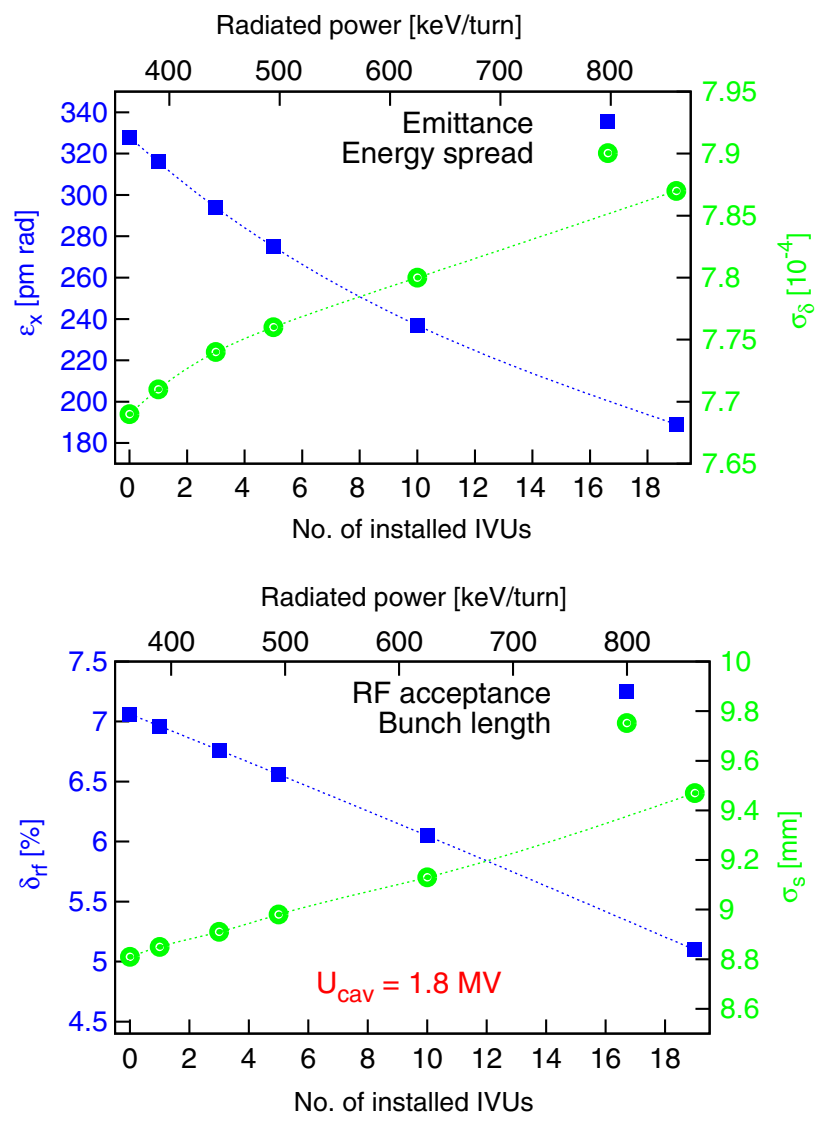

FIG. 1. Beam parameters of the MAX IV $3 \mathrm{GeV}$ storage ring as a function of the number of installed IVUs (for details cf. Sec. II). At fully closed gap each IVU adds $26 \mathrm{keV} /$ turn to the synchrotron radiation losses. Top: zero-current emittance and natural energy spread. Bottom: rf acceptance and zero-current bunch length assuming 1.8 MV overall cavity voltage.

Touschek lifetime $[19,20]$ relies strongly on the 6D emittance: it grows with increasing longitudinal emittance which makes harmonic Landau cavities (LCs) for bunch lengthening attractive [21]. On the other hand, in the ultralow-emittance regime transverse momenta are small compared to the large momentum acceptance (MA) and, therefore, most scattering events do not transfer enough momentum from the transverse to the longitudinal to create Touschek losses. Instead, these events along with IBS lead to a blowup of the 6D emittance. Damping wigglers and IDs reduce the transverse emittance and can therefore increase the Touschek lifetime in ultralow-emittance storage rings. They also achieve this in another way: because their added losses reduce the available cavity overvoltage, they can lengthen the bunches which additionally increases Touschek lifetime. Furthermore, if they increase the energy spread in the bunch, the emittance blowup from IBS is reduced, which in turn also affects the resulting Touschek lifetime. The overall result is that the Touschek lifetime will vary as a function of resulting emittance including IBS as well as bunch lengthening. Since both of these factors are determined by the type of installed IDs and momentary gap settings, the Touschek lifetime can vary during a typical user shift and needs to be calculated for each specific configuration and setting. This shall be investigated in Sec. III.

\section{EMITTANCE AND INTRABEAM SCATTERING}

As established in the Introduction, in MBA lattices with ultralow emittance, the resulting equilibrium emittance depends on the number and type of installed IDs (as well as their gap settings) and-at high stored current-is limited by IBS. This limitation can be recognized in Fig. 2 where the amount of emittance growth from IBS has been calculated assuming the lattice emittance could be varied freely. Regardless if LCs are employed to lengthen bunches or not, below $\approx 100 \mathrm{pm}$ rad the resulting emittance becomes entirely dominated by IBS. The amount of emittance growth caused by IBS itself depends on the bunch charge and 6D bunch emittance. When an ID gap closes the transverse emittance of a bunch can be expected to decrease, while the bunch energy spread can be expected to grow. Furthermore, one must assume that the bunch length can be altered in this process. The result is that because of the gap change, the emittance blowup caused by IBS must be reevaluated.

The 6D tracking code TRACY-3 [22] has been used to calculate equilibrium emittances in all three planes taking into account IBS growth as a function of bunch charge and zero-current emittance. The code has implemented IBS calculations following the Bjorken-Mtingwa [23] as well as the Conte-Martini formalism [24] with the latter having

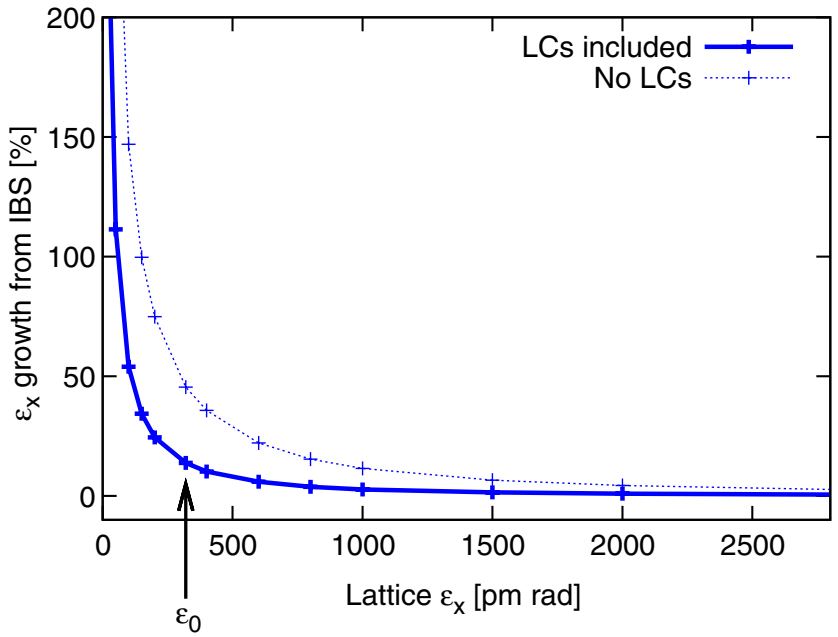

FIG. 2. Horizontal emittance growth caused by IBS at $500 \mathrm{~mA}$ stored current as a function of lattice emittance. It is assumed the latter can be adjusted freely while keeping the energy spread constant. The overall MA has been set to $4.5 \%$ and the vertical emittance is always adjusted to $8 \mathrm{pm}$ rad. The effect of the LCs is shown. The equilibrium emittance $\varepsilon_{0}$ of the MAX IV $3 \mathrm{GeV}$ storage ring bare lattice is indicated. 
been benchmarked against ZAP $[25,26]$. In principle, for each ID and possible gap setting such a calculation has to be carried out. Because of the large number of possible combinations this is impractical. Instead, different lattice configurations of the MAX IV $3 \mathrm{GeV}$ storage ring (where IDs, if included, are assumed to be operated with a fully closed gap) and different settings of emittance coupling have been studied: a bare lattice, a lattice with DWs, and a fully-equipped lattice, i.e., a lattice where an IVU has been installed in every available long straight. For the IVU, it is assumed that a typical device is $3.7 \mathrm{~m}$ long, has a magnetic period of $18.5 \mathrm{~mm}$, and an effective magnetic field of $1.11 \mathrm{~T}$ [27] (adding $26 \mathrm{keV} /$ turn losses). For the lattice with DWs, a total of four DWs has been added to the lattice. Each DW is $2 \mathrm{~m}$ long, has a period length of $80 \mathrm{~mm}$ and a peak magnetic field strength of $2.22 \mathrm{~T}$ [6] (adding $53 \mathrm{keV} /$ turn losses). The emittance for the lattice with DWs is almost identical to that of a moderately IDequipped lattice, i.e., a lattice where 10 IVUs have been installed. The calculations have been performed assuming two different settings of coupling [28]: one corresponding to the baseline $8 \mathrm{pm}$ rad vertical emittance (1 $\AA$ diffraction limit) and one corresponding to a reduced coupling in order to increase photon brightness [29]. The results of these calculations are displayed in Table I. As IDs are added to the storage ring, the emittance decreases thus increasing the charge density and intensifying IBS. Note that since the rf cavities were assumed set to maximum accelerating voltage, the rf acceptances and natural bunch lengths vary between different configurations.

In addition to comparing zero-current emittances to emittances assuming $500 \mathrm{~mA}$ stored beam (in an even fill, i.e., $5 \mathrm{nC}$ charge per bunch), results are also displayed where all bunches are assumed to be stretched by LCs [30]. Since LCs dilute the charge density in the bunch, they weaken IBS: even in a fully ID-equipped ring emittances of roughly $200 \mathrm{pm}$ rad can still be achieved. Although LCs are employed in several 3rd generation storage rings to

TABLE I. Emittance (in pm rad) in the MAX IV $3 \mathrm{GeV}$ storage ring for different settings of coupling and three lattice configurations: bare lattice, lattice with damping wigglers (equivalent to a moderately ID-equipped ring), and a fully ID-equipped ring. Emittance blowup from IBS with and without bunch lengthening from LCs was calculated assuming $500 \mathrm{~mA}$ stored current and rf cavities set to maximum voltage.

\begin{tabular}{|c|c|c|c|c|}
\hline & $\varepsilon_{y}$ & $\begin{array}{l}\text { Zero-current } \\
\varepsilon_{x}\end{array}$ & $\begin{array}{c}\text { IBS } \\
\varepsilon_{x} \\
\end{array}$ & $\begin{array}{l}\text { IBS and LCs } \\
\varepsilon_{x} \\
\end{array}$ \\
\hline \multirow[t]{2}{*}{ Bare } & 8 & 320 & 466 & 364 \\
\hline & 2 & 326 & 552 & 404 \\
\hline \multirow[t]{2}{*}{ DWs } & 8 & 226 & 354 & 264 \\
\hline & 2 & 232 & 436 & 302 \\
\hline \multirow[t]{2}{*}{ Loaded } & 8 & 179 & 292 & 213 \\
\hline & 2 & 185 & 365 & 247 \\
\hline
\end{tabular}

increase lifetime, they will be indispensable in the MAX IV $3 \mathrm{GeV}$ storage ring to ensure ultralow emittance is preserved at full stored current.

The examples in Table I illustrate the large impact IDs have on the resulting emittance in ultralow-emittance rings based on MBA lattices. They also indicate that the emittance in such rings will not remain constant during user operation, but will vary as ID gaps are changed. In principle, one can consider operating DWs in order to counteract such emittance variations and ensure constant emittance during user shifts. However, since ID gaps are rarely ramped across their full range continuously during user shifts and since gap motion is usually not correlated between different beam lines, one can expect that emittance variations during user shifts should remain limited even without compensation by DWs.

It is also noteworthy that lowering the accelerating voltage in the main rf cavities can result in yet lower emittance. For the results presented in Table I, all cavities were assumed to be set to maximum accelerating voltage. This, however, means that the resulting bunch length is short and IBS becomes strongest. If the rf voltage is moderately reduced the bunch length increases limiting IBS. This shall be investigated in the next section.

\section{MOMENTUM ACCEPTANCE AND LIFETIME}

Achieving good Touschek lifetime depends on ensuring large MA. If the lattice MA is sufficient throughout the entire achromat and the rf cavities deliver enough accelerating voltage, the overall MA can become very large compared to the transverse momenta of the electrons in an ultralow-emittance storage ring. This ensures that Touschek lifetime in ultralow-emittance rings remains manageable despite the very high charge density in the bunch, and in fact, can improve when further lowering the transverse emittance. Therefore, ensuring large lattice MA has been one of the main goals of the nonlinear optics optimization for the MAX IV $3 \mathrm{GeV}$ storage ring [6,15]. The requirement for the lattice MA was to match the provided $\mathrm{rf}$ acceptance [31] as well as possible. Figure 3 shows the lattice MA of the MAX IV $3 \mathrm{GeV}$ storage ring bare lattice derived from 6D tracking with TRACY-3 for one synchrotron oscillation period. Tracking reveals that the lattice MA exceeds the minimum requirement of $4.5 \%$ throughout the entire achromat.

\section{A. Touschek lifetime}

With the lattice MA established, Touschek lifetime can be calculated as a function of rf acceptance, bunch charge and $6 \mathrm{D}$ emittance. This has been carried out with TRACY-3 [32] using the MAX IV $3 \mathrm{GeV}$ storage ring as an example. Figure 4 shows results and confirms that the Touschek lifetime is high despite the ultralow emittance. Besides the behavior well known from 3rd generation light sources 


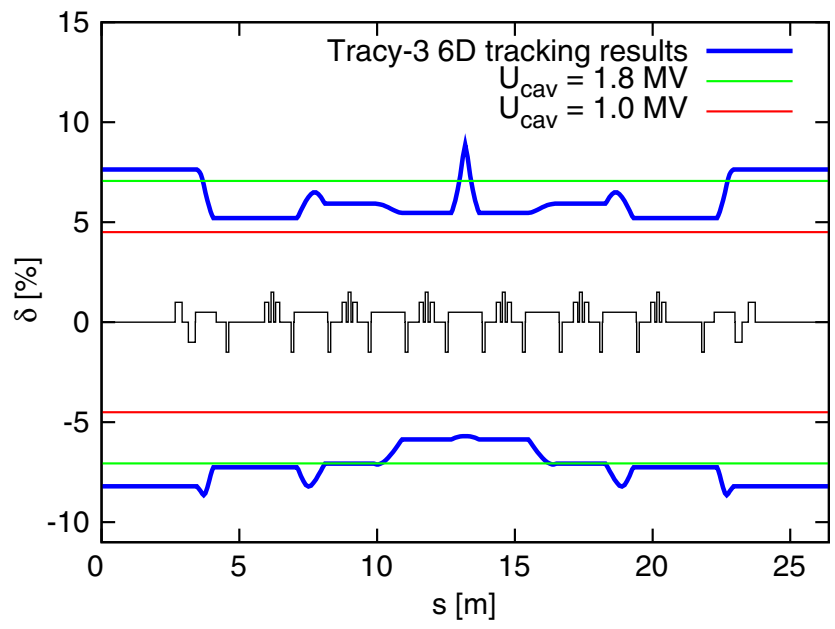

FIG. 3. Lattice MA for one achromat of the MAX IV $3 \mathrm{GeV}$ storage ring. The solid blue line shows lattice MA from 6D tracking with TRACY-3 using actual vacuum chamber apertures. For comparison, the bare lattice rf acceptance is shown as well: cavities at maximum voltage $1.8 \mathrm{MV}(7.1 \% \mathrm{rf}$ acceptance $)$ and at 1.0 MV (4.5\% rf acceptance). The magnetic lattice is indicated at the center.

(above $\approx 1000 \mathrm{pm} \mathrm{rad}$ ), one can recognize the entirely different behavior of ultralow-emittance rings (below $\approx 500 \mathrm{pm} \mathrm{rad}$ ) where a sharp increase of Touschek lifetime occurs when the emittance is lowered. The MAX IV $3 \mathrm{GeV}$ storage ring is clearly operated in this regime where Touschek lifetime can be expected to improve as IDs are added.

To investigate this, Touschek lifetime is calculated for different ID configurations of the storage ring as well as for

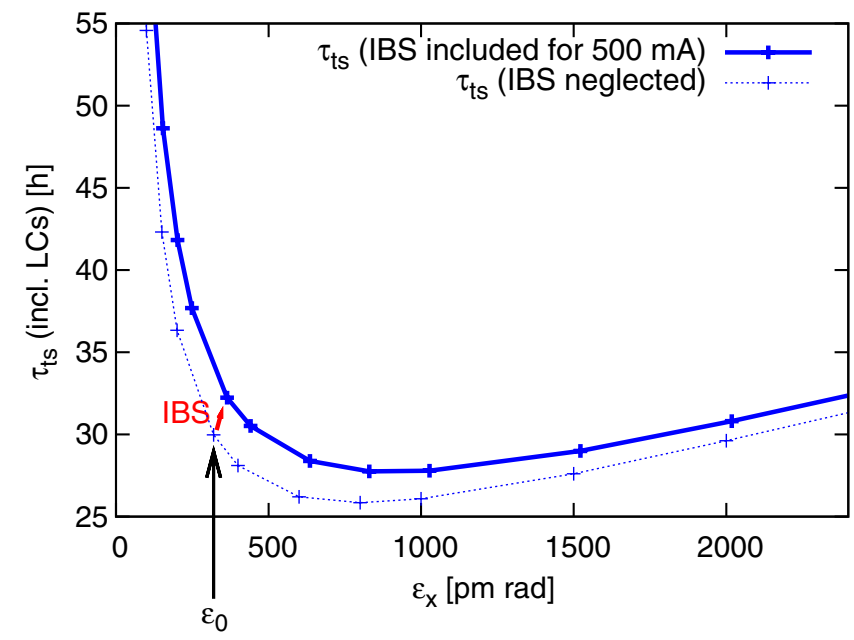

FIG. 4. Touschek lifetime (including the effect of LCs) from 6D tracking with TRACY-3 as a function of equilibrium emittance assuming the lattice emittance could be adjusted freely while keeping the energy spread constant. The overall MA has been set to $4.5 \%$ while the vertical emittance is adjusted to $8 \mathrm{pm}$ rad. The effect of IBS at $500 \mathrm{~mA}$ stored current vs no IBS is displayed. different settings of coupling in a self-consistent manner. In a first step, the zero-current emittance for a given lattice including IDs and rf cavity settings is calculated from the radiation integrals. From $6 \mathrm{D}$ tracking including vacuum apertures and possible imperfections such as field and multipole errors as well as misalignments, the local lattice MA around the ring is then derived. If LCs are employed, the $6 \mathrm{D}$ bunch emittance has to be updated to reflect the bunch lengthening. At this point, the IBS growth rates for a specific bunch charge and emittance are calculated. Iteration then allows finding the new equilibrium emittance. Finally, using the updated emittance, the Touschek lifetime can be calculated by integrating around the entire ring. Results of such tracking studies with TRACY-3 are displayed in Table II.

For a given setting of vertical emittance, there is a slight increase of Touschek lifetime when going from the bare lattice to the moderately ID-equipped ring. This is the result of two competing effects. The rf acceptance of the bare lattice case is larger increasing its Touschek lifetime. On the other hand, the bare lattice has a larger emittance which leads to lower lifetime (cf. Fig. 4). When going from the moderately ID-equipped lattice to the fully loaded ring, there is a substantial decrease of Touschek lifetime. This is again the result of two competing effects. The additional emittance reduction leads to a further Touschek lifetime increase. On the other hand, the rf acceptance is now considerably reduced $(5.1 \%$ for the loaded ring vs the $6.1 \%$ of the moderately ID-equipped ring), so that the overall MA is more heavily dominated by the rf acceptance.

Since the lattice MA is large, the available rf acceptance has a strong influence on the Touschek lifetime. This is demonstrated in Fig. 5. Above 1.2 MV (corresponding to $5.2 \% \mathrm{rf}$ acceptance) the lattice acceptance begins to dominate the overall MA and hence the lifetime starts to taper off. As the cavity voltage is further increased, the bunch length continues to reduce. Beyond 1.4 MV

TABLE II. Touschek lifetime (in hours) in the MAX IV $3 \mathrm{GeV}$ storage ring for different settings of coupling and three lattice configurations (identical to those used in Table I). A stored current of $500 \mathrm{~mA}$ and $\mathrm{rf}$ cavities set to maximum voltage were assumed. The last column shows results where imperfections (misalignments, field and multipole errors) and reduced vertical acceptance from IVUs have been included in the model.

\begin{tabular}{lcccc}
\hline \hline & $\begin{array}{c}\varepsilon_{y} \\
{[\mathrm{pm} \text { rad }]}\end{array}$ & $\begin{array}{c}500 \mathrm{~mA} \\
\text { no LCs }\end{array}$ & $\begin{array}{c}500 \mathrm{~mA} \\
\text { including LCs }\end{array}$ & $\begin{array}{c}\text { Including errors } \\
\text { and narrow gaps }\end{array}$ \\
\hline Bare & 8 & 17.4 & 87.1 & 64.3 \\
& 2 & 9.6 & 45.9 & 40.7 \\
DWs & 8 & 20.5 & 114.3 & 66.2 \\
& 2 & 10.4 & 56.1 & 48.7 \\
Loaded & 8 & 11.7 & 65.0 & 37.7 \\
& 2 & 5.8 & 31.4 & 27.3 \\
\hline
\end{tabular}

${ }^{a}$ Narrow gaps have not been included in the bare lattice case. 


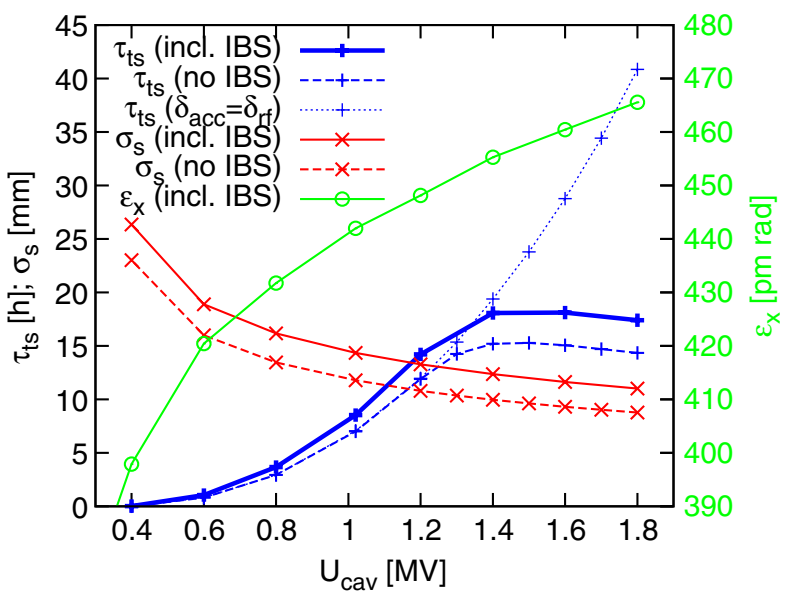

FIG. 5. Touschek lifetime, bunch length, and horizontal emittance in the MAX IV $3 \mathrm{GeV}$ storage ring bare lattice as functions of the rf cavity voltage. The stored current was assumed to be $500 \mathrm{~mA}$ with the vertical emittance always adjusted to $8 \mathrm{pm} \mathrm{rad}$. Bunch lengthening from LCs has not been included.

(corresponding to $5.9 \% \mathrm{rf}$ acceptance) this results in a decrease of Touschek lifetime. However, a lifetime gain of up to 2-3 hours compared to the zero-current case can also be recognized as a result of the charge density dilution caused by IBS.

The example in Fig. 5 does not include bunch lengthening from LCs which are expected to be in use during user operation. Because LCs reduce the emittance blowup from IBS, the Touschek lifetime can be increased further (cf. Fig. 4) compared to the result from bunch lengthening alone. Figure 6 shows how Touschek lifetime increases with the bunch length while the emittance decreases.

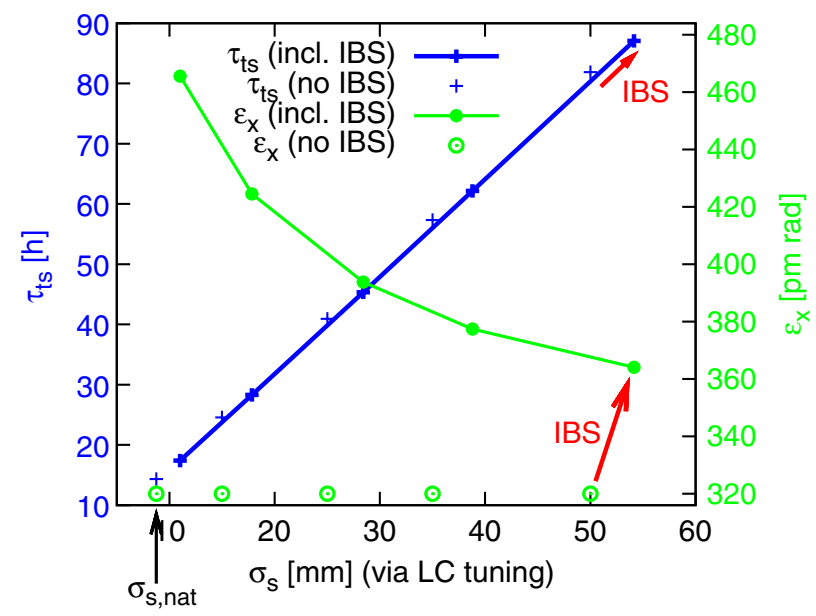

FIG. 6. Touschek lifetime and horizontal emittance as functions of bunch length which is adjusted by tuning the LCs. The stored current was assumed to be $500 \mathrm{~mA}$ with the vertical emittance always set to $8 \mathrm{pm}$ rad. The calculated Touschek lifetime is based on resulting overall MA from tracking and actual vacuum apertures. The emittance blowup and bunch length increase from IBS as well as the associated lifetime increase are indicated.

\section{B. Overall lifetime}

To calculate the overall lifetime, the gas scattering lifetimes have to be added inversely to the Touschek lifetime. However, in order to model a realistic machine, the Touschek lifetime must also include the effect of imperfections which reduce the off-momentum dynamic aperture, as well as additional acceptance limitations imposed by the closed gaps of IVUs or by narrow-gap ID chambers. Results of such effects on the MAX IV $3 \mathrm{GeV}$ storage ring are displayed in the last column of Table II. The vertical apertures in the long straights were reduced to $\pm 2 \mathrm{~mm}$ over a $4 \mathrm{~m}$ section of every long straight to model the acceptance limitation imposed by an IVU with closed gap. For the imperfections, 100 seeds were studied with field and multipole errors as well as misalignments. After alignment errors had been applied, orbit correction was simulated.

When all these effects are included, a Touschek lifetime of $27.3 \pm 2.1 \mathrm{~h}$ results even in the case of reduced $\mathrm{rf}$ acceptance (fully ID-equipped ring) and reduced vertical emittance. The uncertainty corresponds to one standard deviation of the error seeds. The gas scattering lifetimes in the MAX IV $3 \mathrm{GeV}$ storage ring including in-vacuum IDs have been estimated at roughly 25 hours (elastic) and 56 hours (inelastic) where the latter has been calculated assuming a MA of only 4.5\% [14]. The total lifetime should therefore always be above $10 \mathrm{~h}$ which is compatible with the foreseen top-up injection scheme with one top-up injection every few minutes ensuring a top-up deadband of about $0.5 \%$ [33]. For a moderately ID-equipped ring a Touschek lifetime beyond $49 \mathrm{~h}$ can be expected depending on the choice of coupling. This corresponds to a total lifetime above $12 \mathrm{~h}$. It is interesting to note that the lifetime in such a configuration is no longer Touschek-dominated as is commonly the case in 3rd generation storage rings.

\section{CONCLUSIONS}

Modern ultralow-emittance storage rings at medium energy are characterized by strong IBS and potentially large Touschek lifetime if sufficient MA is provided by the lattice and rf system. Fully self-consistent tracking simulations are required to model the intricate interplay between the transverse and longitudinal emittances as a function of the bunch charge. Unlike existing 3rd generation storage rings, key parameters of these light sources will not remain constant during user shifts as they depend on continually varying ID gaps and the choice of rf cavity settings. In these rings one could therefore consider adding DWs specifically to enable adjusting the emittance to ensure constant electron beam dimensions during user shifts.

In these ultralow-emittance storage rings, LCs are both essential and versatile tools: besides mitigation of multibunch instabilities via Landau damping and bunch lengthening, they also preserve the ultralow emittance. As the examples in this paper show, good lifetime can be achieved despite the very low emittance through the application of 
bunch lengthening LCs. Landau cavities are, however, also required in order to limit emittance growth from IBS thus preventing saturation of the achievable photon brightness at high bunch charge. Note also, that even in a fully diffraction-limited regime, the energy spread blowup caused by IBS can reduce the spectral brightness.

To further reduce the emittance and increase the Touschek lifetime of ultralow-emittance rings, one can contemplate adding additional DWs. Specifically, one could suggest starting to operate such light sources with DWs in all unoccupied user straights, removing a DW only when a user ID is ready to be installed in its place. Apart from the high cost (not only the initial cost of the DWs, but also the increased running cost that results from the high rf power consumption) of such a strategy, one should carefully analyze the resulting photon brightness. Meticulous DW design is required if the added energy spread shall not spoil any brightness gains from the reduced emittance.

Finally, it should be acknowledged that the strong emittance blowup from IBS is mainly an issue in medium-energy rings. Increasing the storage ring energy reduces the impact of IBS. The strong IBS in mediumenergy ultralow-emittance rings is further compounded by low values of emittance coupling. The prospect of diffraction-limited storage rings operated at full coupling with round beams thus presents an interesting alternative.

\section{ACKNOWLEDGMENTS}

The author would like to express his gratitude to Johan Bengtsson (NSLS-II) for supplying the TRACY-3 libraries and the IBS routines along with much support. Thanks also go to Åke Andersson (MAX IV) for carrying out ZAP calculations used for benchmarking. Finally, the author thanks Les Dallin (CLS) and Andreas Streun (SLS) for their suggestions and for proofreading of the manuscript.

[1] D. Einfeld and M. Plesko, Proc. SPIE Int. Soc. Opt. Eng. 2013, 201 (1993).

[2] D. Einfeld and M. Plesko, Nucl. Instrum. Methods Phys. Res., Sect. A 335, 402 (1993).

[3] W. Joho, P. Marchand, L. Rivkin, and A. Streun, in Proceedings of the Fourth European Particle Accelerator Conference EPAC 94, London, England (World Scientific, River Edge, NJ, 1994), p. 627.

[4] D. Einfeld, J. Schaper, and M. Plesko, in Proceedings of the Particle Accelerator Conference, Dallas, TX, 1995 (IEEE, New York, 1995), p. 177.

[5] D. Kaltchev, R. V. Servranckx, M. K. Craddock, and W. Joho, in Proceedings of the Particle Accelerator Conference, Dallas, TX, 1995 (IEEE, New York, 1995), p. 2823.

[6] S. C. Leemann, A. Andersson, M. Eriksson, L.-J. Lindgren, E. Wallén, J. Bengtsson, and A. Streun, Phys. Rev. ST Accel. Beams 12, 120701 (2009).
[7] L. Liu et al., in Proceedings of the 4th International Particle Accelerator Conference, IPAC-2013, Shanghai, China, 2013 (JACoW, Shanghai, China, 2013), p. 1874.

[8] L. Faravacque et al., in Proceedings of the 4th International Particle Accelerator Conference, IPAC-2013, Shanghai, China, 2013 (JACoW, Shanghai, China, 2013), p. 79.

[9] Y. Shimosaki et al., in Proceedings of the 3rd International Particle Accelerator Conference, New Orleans, LA, 2012 (IEEE, Piscataway, NJ, 2012), p. 1185.

[10] M. Borland, V. Sajaev, and Y. Sun, in Proceedings of the 25th Particle Accelerator Conference, PAC-2013, Pasadena, CA, 2013 (IEEE, New York, 2013), p. 249.

[11] H. Tarawneh et al., in Proceedings of the 25th Particle Accelerator Conference, PAC-2013, Pasadena, CA, 2013 (IEEE, New York, 2013), p. 288.

[12] R. Hettel and M. Borland, in Proceedings of the 25th Particle Accelerator Conference, PAC-2013, Pasadena, CA, 2013 (IEEE, New York, 2013), p. 19.

[13] M. Eriksson et al., Proceedings of the 24th Particle Accelerator Conference, PAC-2011, New York, 2011 (IEEE, New York, 2011), p. 737.

[14] MAX IV Detailed Design Report, available for download at http://www.maxlab.lu.se/maxlab/max4/index.html, 2010.

[15] S. C. Leemann and A. Streun, Phys. Rev. ST Accel. Beams, 14, 030701 (2011).

[16] It is assumed here that the achromat optics can be matched to an ID and its gap setting [27]. Therefore, the emittance change after a gap variation is the result of a change of radiated power, not of machine functions.

[17] A. Piwinski, in Proceedings of the 9th International Conference on High Energy Accelerators, Stanford, 1974, p. 405.

[18] J. Le Duff, CERN Report No. 89-01, 1989, p. 114.

[19] C. Bernadini, G. Corazza, G. Di Giugno, G. Ghigo, J. Haissinski, P. Marin, R. Querzoli, and B. Touschek, Phys. Rev. Lett., 10, 407 (1963).

[20] H. Bruck, Accélérateurs Circulaires de Particules (Presses Universitaires de France, Paris, 1966).

[21] J. M. Byrd and M. Georgsson, Phys. Rev. ST Accel. Beams 4, 030701 (2001).

[22] J. Bengtsson, Tracy-2 User's Manual (unpublished).

[23] J. D. Bjorken and S. K. Mtingwa, Part. Accel. 13, 115 (1983).

[24] M. Conte and M. Martini, Part. Accel. 17, 1 (1985).

[25] M. S. Zisman, S. Chattopadhyay, and J. J. Bisognano, ZAP User's Guide, LBL 21270, 1986.

[26] Several attempts have been made to validate IBS theory in existing electron storage rings, e.g., [34-36]. Reasonably good agreement between theory and experimental data has been found, however, deviations start to grow when the strong IBS regime is reached. Once the MAX IV $3 \mathrm{GeV}$ storage ring becomes operational in 2016, it should present an interesting opportunity to investigate this regime more closely.

[27] E. Wallén and S. C. Leemann, in Proceedings of the 24th Particle Accelerator Conference, PAC-2011, New York, 2011 (IEEE, New York, 2011), p. 1262.

[28] The underlying assumption is that betatron coupling and spurious vertical dispersion determine the achievable vertical emittance, and that the latter can therefore be adjusted with the available skew quadrupoles. 
[29] S. C. Leemann and M. Eriksson, in Proceedings of the 25th Particle Accelerator Conference, PAC-2013, Pasadena, CA, 2013 (IEEE, New York, 2013), pp. 243-245.

[30] The passive LCs of the MAX IV $3 \mathrm{GeV}$ storage ring can stretch bunches beyond the natural bunch length by slightly more than a factor 5 [37].

[31] Because the MAX IV storage rings operate with $100 \mathrm{MHz}$ main rf, large rf acceptance can be ensured with moderate cavity voltage. This leads to low rf power requirements and hence lower running cost despite providing considerable bucket height.

[32] C. Montag, J. Bengtsson, and B. Nash, in Proceedings of the 22nd Particle Accelerator Conference, PAC-2007, Albuquerque, NM (IEEE, New York, 2007), p. 4375.
[33] S. C. Leemann, Phys. Rev. ST Accel. Beams 15, 050705 (2012).

[34] K. L. F. Bane, H. Hayano, K. Kubo, T. Naito, T. Okugi, and J. Urakawa, Phys. Rev. ST Accel. Beams 5, 084403 (2002).

[35] M. P. Ehrlichman et al., Phys. Rev. ST Accel. Beams 16, 104401 (2013).

[36] F. Antoniou and Y. Papaphilippou, in 3rd Low Emittance Rings Workshop, Oxford, 2013, https://indico.cern.ch/ event/247069/session/0/contribution/6/material/slides/1 .pdf.

[37] A. Andersson et al., in Proceedings of the 2nd International Particle Accelerator Conference, San Sebastián, Spain (EPS-AG, Spain, 2011), p. 193. 